\title{
Spectroscopic Detection of the LiHe Molecule
}

\author{
Naima Tariq, Nada Al Taisan, Vijay Singh, and Jonathan D. Weinstein* \\ Department of Physics, University of Nevada, Reno, Nevada 89557, USA
}

(Received 27 December 2012; published 8 April 2013)

\begin{abstract}
We use cryogenic helium buffer-gas cooling to form large densities of lithium atoms in a high-density helium gas, from which LiHe molecules form by three-body recombination. These weakly bound van der Waals molecules are detected spectroscopically, and their binding energy is measured from their equilibrium thermodynamic properties.
\end{abstract}

DOI: 10.1103/PhysRevLett.110.153201

PACS numbers: 34.50.Lf, 33.15.Fm, 33.20.-t

van der Waals (vdW) molecules, which are bound by long-range dispersion interactions, represent the most weakly bound form of molecular matter [1]. Of the diatomic vdW molecules, those containing helium - the most chemically "inert" of the noble gases-are the most weakly bound. There is much theoretical interest in the study of diatomic molecules involving helium, including questions over their existence [2-4]. To date, the only ground-state helium diatomic molecule that has been directly detected in the gas phase is $\mathrm{He}_{2}$ [5-7].

Kleinekathöfer et al. [3] predict that for all ${ }^{4} \mathrm{He}$-alkali diatomic molecules, there exists a single bound rovibrational state in the $X^{2} \Sigma$ ground electronic state. ${ }^{7} \mathrm{Li}^{4} \mathrm{He}$ is predicted to have a bond length of $\langle r\rangle \sim 28 \AA$, and a binding energy of $0.0039 \mathrm{~cm}^{-1}$ [3]. These numbers are comparable to ${ }^{4} \mathrm{He}_{2}$ [6], despite the significantly smaller well depth of ${ }^{7} \mathrm{Li}^{4} \mathrm{He}$ [8].

Experimentally, vdW molecules have been investigated through a variety of techniques. Supersonic expansions have produced argon- and neon-based vdW molecules for spectroscopic studies as well as helium bound to large molecules [9]. Excited-state helium vdW molecules have been observed in liquid helium [10], in dense helium gas [11-13], and in superfluid helium nanodroplets [14,15].

Brahms et al. observed that helium buffer-gas cooling would provide a favorable environment for forming ground-state helium vdW molecules and demonstrated indirect evidence for the formation of $\mathrm{Ag}^{3} \mathrm{He}$ [16]. Following this work, we have prepared high densities of cold lithium atoms in a cryogenic helium gas, in order to create LiHe molecules through three-body recombination,

$$
\mathrm{Li}+\mathrm{He}+\mathrm{He} \leftrightarrow \mathrm{LiHe}+\mathrm{He} .
$$

We have spectroscopically detected the LiHe molecules produced, measured their equilibrium properties, and measured the binding energy of the ground state.

In thermal equilibrium, the expected density of LiHe is given by

$$
n_{\mathrm{LiHe}}=n_{\mathrm{Li}} n_{\mathrm{He}}\left(\frac{h^{2}}{2 \pi \mu k_{B} T}\right)^{3 / 2} \sum_{i} g_{i} e^{\epsilon_{i} / k_{B} T},
$$

where $n$ is the density of the given species, $\mu$ is the reduced mass, $T$ is the temperature, and $g_{i}$ and $\epsilon_{i}$ are the degeneracies and binding energies of the LiHe bound states [16]. Clearly, the formation of detectable quantities of LiHe is favored by high lithium and helium densities and low temperatures. All three are achieved with cryogenic helium buffer-gas cooling of atomic lithium produced by laser ablation [17].

We note that the exceptionally low binding energy of lithium to helium makes the formation of LiHe less favorable than many other helium vdW molecules [18]. We chose to search for LiHe due to our group's prior experience working with atomic lithium [17], prior theoretical work on the structure of the LiHe molecule [3,19-22], and because atomic lithium's $D$ transition is easily accessed with diode lasers.

The experiment is conducted in a cryogenic cell, identical to the apparatus described in Ref. [17]. The cell temperature is monitored by a ruthenium oxide resistor and a silicon diode. The helium density in the cell is determined from a room-temperature pressure gauge connected to the cell through a thin tube and from the cell temperature. We correct for the thermomolecular pressure ratio with the Weber-Schmidt equation [23]. The uncertainty in the helium density is approximately $\pm 20 \%$.

Gas-phase $\mathrm{Li}$ is produced by laser ablation of a solid target of $99.9 \%$ pure $\mathrm{Li}$ (of natural isotopic abundances) with a frequency-doubled $\mathrm{Nd}$ :YAG laser. The $\mathrm{Li}$ atoms are detected by laser absorption spectroscopy on the $D_{1}$ transition at $671 \mathrm{~nm}[24,25]$. Due to the high atomic densities employed in the experiment, we typically measure the atomic lithium via the less-abundant ${ }^{6} \mathrm{Li}$ isotope. The probe power is $\ll 1 \mu \mathrm{W}$, with a beam diameter of a few $\mathrm{mm}$. (At powers $\gtrsim 1 \mu \mathrm{W}$, we observe optical pumping of the $\mathrm{Li}$ atoms, which makes accurate measurements of density difficult.) The helium buffer-gas density ranges from $2 \times 10^{17}$ to $1 \times 10^{18} \mathrm{~cm}^{-3}$.

We are able to produce Li densities up to $10^{11} \mathrm{~cm}^{-3}$, with ablation energies of tens of mJ. The ablation pulse momentarily increases the temperature of the gas [26], so we directly measure the translational temperature of the gas through spectroscopy of the atomic ${ }^{6} \mathrm{Li}$; under our 
conditions, Doppler broadening is observed to be the dominant broadening mechanism.

Typical Li lifetimes in the cell are on the order of $10^{-1} \mathrm{~s}$. We note that the lithium density as a function of time is not described by simple exponential decay; this is commonly seen in buffer-gas cooling experiments at high helium density [27]. To avoid complications due to this complex behavior, we measure $\mathrm{Li}$ and $\mathrm{LiHe}$ simultaneously.

LiHe molecules are detected by laser induced fluorescence (LIF). The LiHe probe beam power is on the order of a few $\mathrm{mW}$, with a beam diameter of a few $\mathrm{mm}$. We note that the LiHe probe beam is spatially overlapped with the $\mathrm{Li}$ probe beam to within $3 \mathrm{~mm}$. This overlap is important, as the density distribution of $\mathrm{Li}$ throughout the cell is expected to be nonuniform [17]. The LIF signal is observed using a $\mathrm{Si}$ photodiode. To calibrate the frequency of the LiHe probe beam, part of the beam is split off and sent through a $100 \mathrm{~mm}$-long iodine cell at $300 \mathrm{~K}[28,29]$, and another beam is sent through a Fabry-Perot cavity so that the laser scan can be linearized. The LiHe LIF and the iodine signals are shown in Fig. 1; the frequency is calibrated from the iodine signal. We note that we see no evidence of pressure shifts (or pressure broadening) over the density range explored in this experiment, to within our experimental error of $0.004 \mathrm{~cm}^{-1}$ in absolute frequencies.

The spectrum is quite simple, due to the structure of the ground state of $\mathrm{LiHe}$, which is predicted to have only a single bound rovibrational state [3]. As seen in Fig. 1, we observe two pairs of lines separated by $0.1774 \pm$ $0.0025 \mathrm{~cm}^{-1}$. The splitting of each pair is the same to within our experimental error, with an average value of $0.0276 \pm 0.0004 \mathrm{~cm}^{-1}$.

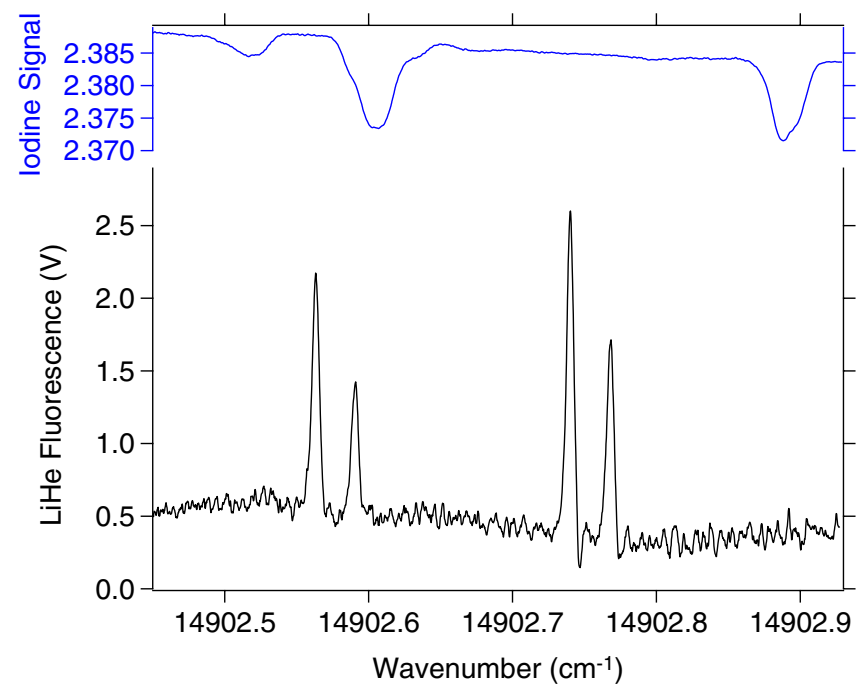

FIG. 1 (color online). Fluorescence spectrum of LiHe molecules. The LiHe fluorescence is plotted against the lower axis; the iodine signal used for frequency calibration is shown on the upper axis. The graph is an average of multiple spectra, taken at various times ranging from 16 to $40 \mathrm{~ms}$ after the ablation pulse, temperatures from 2.0 to $2.5 \mathrm{~K}$, and a helium density of $7 \times 10^{17} \mathrm{~cm}^{-3}$.
We attribute the larger splitting to the excited-state structure and the smaller splitting of each pair to the hyperfine structure of the ground state, as shown in Fig. 2. We note that the measured ground-state hyperfine splitting is within $2 \sigma$ of the atomic ${ }^{7} \mathrm{Li}$ ground-state hyperfine splitting. Due to the extremely weak binding energy of the molecule, we would expect the hyperfine splitting to be unchanged at the level of accuracy of our measurement. From this hyperfine splitting and the rotational structure of the excited state (discussed below), we identify the molecule as ${ }^{7} \mathrm{Li}^{4} \mathrm{He}$.

To verify the assignment of the transitions, we calculated the structure of the highest-lying bound vibrational level of the first excited state of LiHe. The molecular potentials that correlate to the $\mathrm{He}\left({ }^{1} S\right) \mathrm{Li}\left({ }^{2} P\right)$ excited state are $A^{2} \Pi$ and $B^{2} \Sigma$. We construct simple model potentials for the $A^{2} \Pi$ and $B^{2} \Sigma$ states of LiHe using the Buckingham potential formula for each,

$$
V(r)=C_{1} e^{-C_{2} r}-\frac{C_{6}}{r^{6}} .
$$

The long-range coefficients $C_{6}$ are taken from the calculations of Zhu et al. [20], and $C_{1}$ and $C_{2}$ are determined by fitting to the potentials calculated by Behmenburg et al. [19].

We first performed single-channel calculations of the energy levels of the $A^{2} \Pi$ and $B^{2} \Sigma$ model potentials. No bound states were found for the $B$ state, whereas the $A$ state supported multiple vibrational levels. Unfortunately, these single-channel calculations (which omit mixing of the $A$ and $B$ states and do not include the lambda doubling) do not reproduce the observed splitting to within the experimental error.

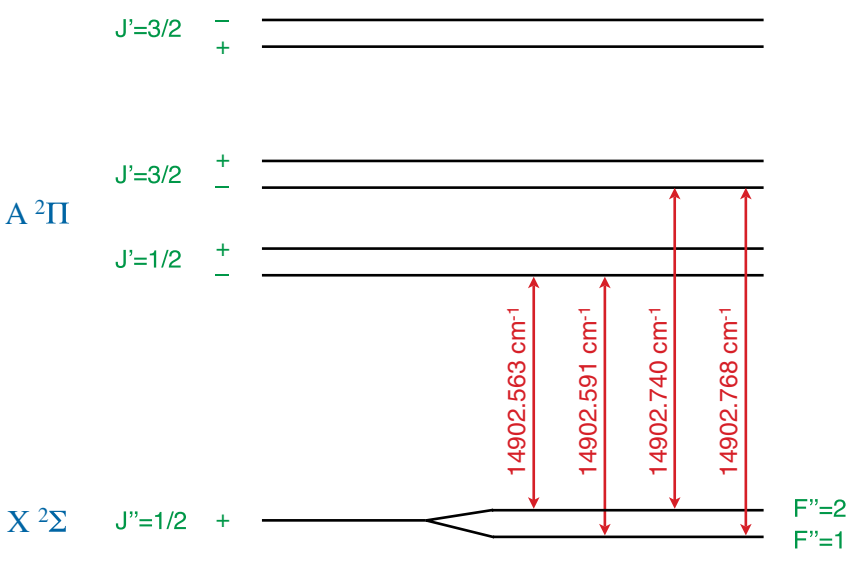

FIG. 2 (color online). Schematic of the relevant LiHe energy levels, showing the ground-state hyperfine structure and the excited-state rotational structure and lambda doubling. Levels are labeled + and - according to their parity. The hyperfine structure in the excited state is not shown, as it is not resolved in our spectra. This is expected, as the hyperfine splitting of the excited state of atomic lithium is small compared to the Doppler broadening. The listed transition frequencies are the experimentally measured values, with experimental error of $\pm 0.004 \mathrm{~cm}^{-1}$. 
Subsequently we performed coupled-channel calculations using the discrete variable representation of Colbert and Miller [30]. The calculation included the $A^{2} \Pi$ and $B^{2} \Sigma$ potentials as described above, the rotational Hamiltonian $\left[1 /\left(2 \mu r^{2}\right)\right]\left(R_{x}^{2}+R_{y}^{2}\right)$ [31], and the finestructure Hamiltonian $A \mathbf{L} \cdot \mathbf{S}$ [31], with the assumption that the fine-structure constant $A$ is unchanged from its atomic value [25]. The $C_{1}$ coefficient of the $A^{2} \Pi$ potential is modified to shift the position of the last energy levels to match our data, with the assumption that the ground-state binding energy is negligible. The coupled-channel calculation gives an energy-level structure as shown in Fig. 2; the parity was determined from the eigenfunctions [32].

We note that once the $A^{2} \Pi$ potential is shifted to give the proper binding energy, the wave function is not sensitive to the inner part of the potential and determined primarily by $C_{6}$ and the binding energy. However, the lambda doubling is sensitive to the exact form of the $B^{2} \Sigma$ potential: both to the choice of which theoretical potential we use [19,21] and whether we fit it to a Buckingham or Lennard-Jones model potential. We find the splitting between the negative parity $J^{\prime}=1 / 2$ and the lower $J^{\prime}=3 / 2$ states is $0.180 \pm$ $0.005 \mathrm{~cm}^{-1}$; the error is estimated from the different results obtained for different $B^{2} \Sigma$ model potentials. This is in quantitative agreement with our measurement. From this agreement, we conclude that we are observing the ${ }^{7} \mathrm{Li}^{4} \mathrm{He}$ molecule and not lithium bound to a helium cluster.

The calculation indicates that a higher-lying $J^{\prime}=3 / 2$ state is also bound, as shown in Fig. 2, which would give rise to a transition roughly $1 \mathrm{~cm}^{-1}$ blueshifted from the $J^{\prime}=1 / 2$ transition. No such transition is observed in the experiment. We suspect this is because the molecular transition is too close to an atomic lithium transition.

We measured the LiHe signal as a function of $n_{\mathrm{Li}}, n_{\mathrm{He}}$, and $T$. The LiHe optical density (OD) as a function of the Li OD is shown in Fig. 3 , at fixed $T$ and $n_{\mathrm{He}}$. We note that the ODs are proportional to the molecular and atomic densities, respectively. The ${ }^{7} \mathrm{Li}$ OD is calculated from the ${ }^{6} \mathrm{Li}$ absorption signal and the known isotopic abundances [24]. To determine the LiHe OD from the LIF signal, we calibrate the LIF with absorption spectroscopy.

As seen in Fig. 3, the LiHe density is linearly dependent on the lithium density, consistent with Eq. (1) and thermal equilibrium. Moreover, the relation between the $\mathrm{Li}$ and $\mathrm{LiHe}$ densities is measured to be independent of the observation time, indicating that the equilibration of the $\mathrm{Li}$ and LiHe populations occurs on a time scale fast compared to diffusion.

Having established a linear relationship between $n_{\mathrm{LiHe}}$ and $n_{\mathrm{Li}}$, we then measure the ratio $n_{\mathrm{LiHe}} / n_{\mathrm{Li}}$ at different helium densities and temperatures. As per Eq. (1), we expect the ratio to increase linearly with the helium density. This is consistent with the data as shown in Fig. 4.

The ratio's dependence on temperature is more interesting, as the form of Eq. (1) allows us to measure the binding

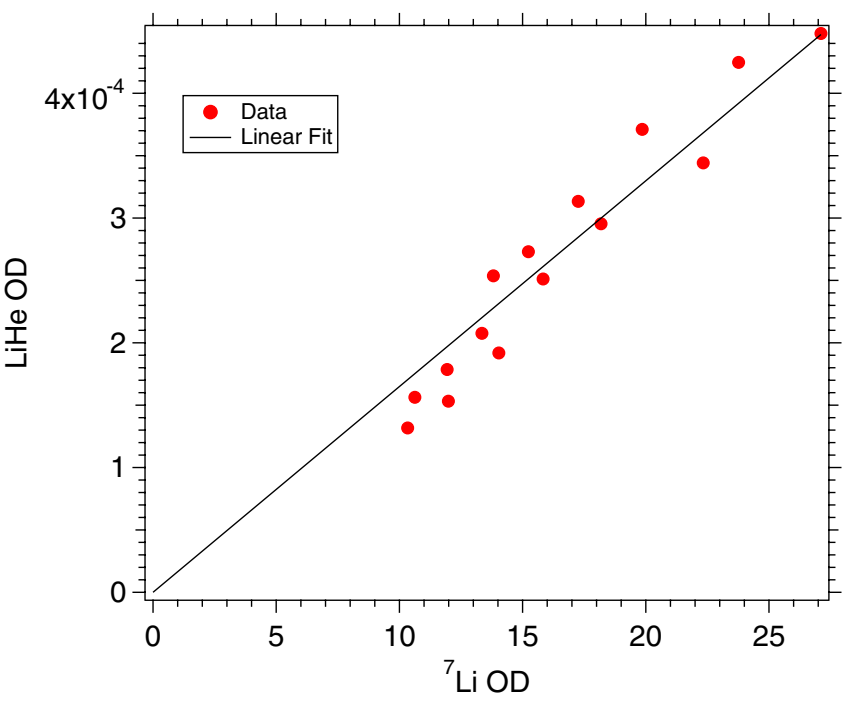

FIG. 3 (color online). LiHe OD plotted as a function of ${ }^{7} \mathrm{Li}$ OD at a constant helium density of $2.3 \times 10^{17} \mathrm{~cm}^{-3}$ and a temperature of $2.45 \pm 0.2 \mathrm{~K}$.

energy. Our measurements of $n_{\mathrm{LiHe}} / n_{\mathrm{Li}}$ as a function of temperature are shown in Fig. 5, over a temperature range from 1.6 to $5 \mathrm{~K}$. The lower end of the temperature range is limited by the base temperature of our cryostat and ablationinduced heating. At higher temperatures, our signal-tonoise ratio suffers due to low $\mathrm{LiHe}$ densities. The data are fit to Eq. (1) with the assumption that there is only one bound rovibrational state: $n_{\mathrm{LiHe}} / n_{\mathrm{Li}} \propto T^{-3 / 2} e^{\epsilon / k_{B} T}$.

By fitting the data in Fig. 5 and similar data taken at other $\mathrm{He}$ densities, we measure the ground-state binding energy of LiHe to be $0.024 \pm 0.025 \mathrm{~cm}^{-1}$. This is consistent with the calculated binding energy $0.0039 \mathrm{~cm}^{-1}$.

Lower temperatures would improve measurements of the binding energy, but buffer-gas cooling temperatures

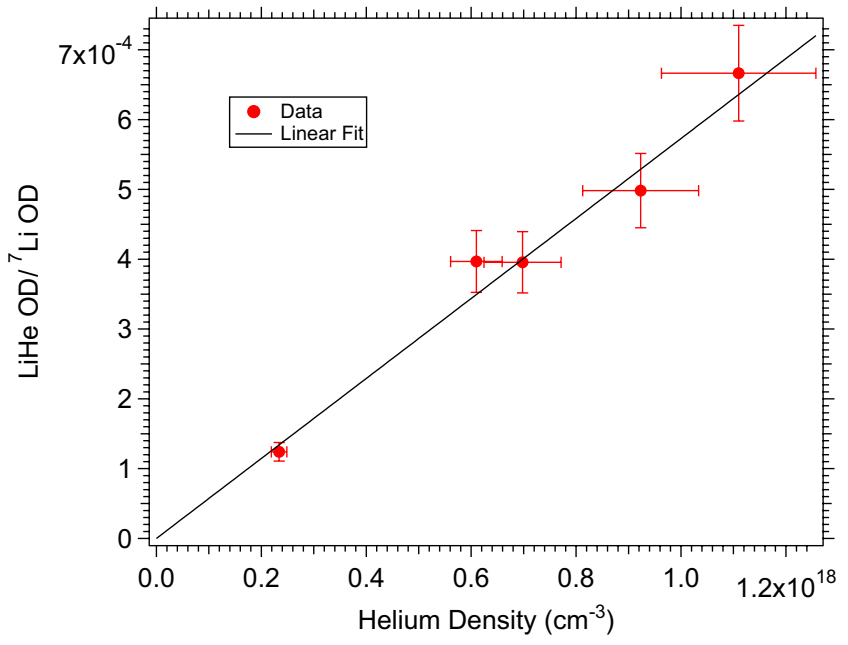

FIG. 4 (color online). The ratio of LiHe OD to ${ }^{7} \mathrm{Li}$ OD as a function of helium density at temperatures from 2.5 to $6 \mathrm{~K}$. Missing from the horizontal error bars is an additional 8\% uncertainty from the uncertainty in our helium fill line diameter. 


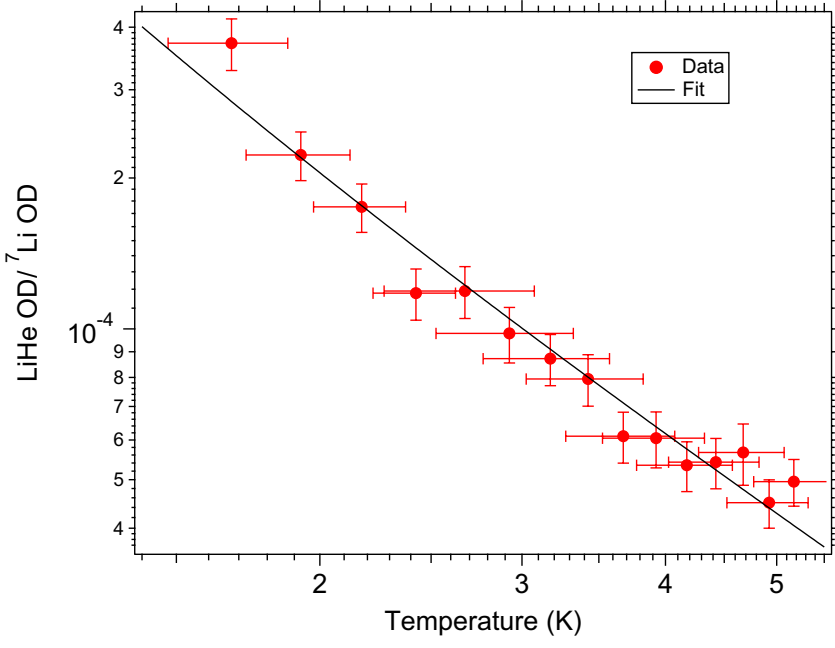

FIG. 5 (color online). The ratio of LiHe OD to ${ }^{7} \mathrm{Li}$ OD as a function of temperature at constant helium density of about $7 \times 10^{17} \mathrm{~cm}^{-3}$. The fit is as discussed in the text.

are limited by the vapor pressure of helium. One might expect that the binding energy could be measured spectroscopically by comparing this data to photoassociation (PA) spectroscopy. Unfortunately, the PA signal is expected to be small under our experimental conditions, and the PA linewidth would be expected to be on the order of $1 \mathrm{~cm}^{-1}$ [33]. While this is convenient for distinguishing the observed bound-bound signal from PA, it precludes making accurate spectroscopic measurements of the binding energy in the current system.

We note that in the measurements presented here, the population of LiHe molecules was in thermal equilibrium. In addition, no optical pumping was observed for the LiHe probe beam intensities employed in this work. This is not surprising, as we operate at high helium densities and the three-body recombination rate coefficient is expected to be very large $[4,18]$. In future work we hope to measure the three-body recombination rate coefficient by using a higher-intensity optical pumping beam to dissociate LiHe molecules and then watch them reform by three-body recombination.

We gratefully acknowledge helpful discussions with Nathan Brahms and Thad G. Walker that inspired this work. We gratefully acknowledge helpful discussions with Eite Tiesinga which guided our calculations of the structure of the excited state of LiHe. This material is based upon work supported by the National Science Foundation under Grant No. PHY 0900190.

*weinstein@physics.unr.edu http://www.physics.unr.edu/xap/

[1] B. L. Blaney and G. E. Ewing, Annu. Rev. Phys. Chem. 27, 553 (1976).
[2] K. T. Tang, J. P. Toennies, and C. L. Yiu, Phys. Rev. Lett. 74, 1546 (1995).

[3] U. Kleinekathöfer, M. Lewerenz, and M. Mladenović, Phys. Rev. Lett. 83, 4717 (1999).

[4] H. Suno and B.D. Esry, Phys. Rev. A 80, 062702 (2009).

[5] F. Luo, G. C. McBane, G. Kim, C. F. Giese, and W. R. Gentry, J. Chem. Phys. 98, 3564 (1993).

[6] R.E. Grisenti, W. Schöllkopf, J.P. Toennies, G. C. Hegerfeldt, T. Köhler, and M. Stoll, Phys. Rev. Lett. 85, 2284 (2000).

[7] W. Schöllkopf and J.P. Toennies, Science 266, 1345 (1994).

[8] J. Yuan and C. D. Lin, J. Phys. B 31, L637 (1998).

[9] U. Even, I. Al-Hroub, and J. Jortner, J. Chem. Phys. 115, 2069 (2001).

[10] J. L. Persson, Q. Hui, Z. J. Jakubek, M. Nakamura, and M. Takami, Phys. Rev. Lett. 76, 1501 (1996).

[11] K. Enomoto, K. Hirano, M. Kumakura, Y. Takahashi, and T. Yabuzaki, Phys. Rev. A 66, 042505 (2002).

[12] K. Hirano, K. Enomoto, M. Kumakura, Y. Takahashi, and T. Yabuzaki, Phys. Rev. A 68, 012722 (2003).

[13] Z. J. Jakubek, Q. Hui, and M. Takami, Phys. Rev. Lett. 79, 629 (1997).

[14] G. Droppelmann, O. Bünermann, C.P. Schulz, and F. Stienkemeier, Phys. Rev. Lett. 93, 023402 (2004).

[15] J. P. Toennies and A. F. Vilesov, Annu. Rev. Phys. Chem. 49, 1 (1998).

[16] N. Brahms, T. V. Tscherbul, P. Zhang, J. Kłos, H. R. Sadeghpour, A. Dalgarno, J. M. Doyle, and T. G. Walker, Phys. Rev. Lett. 105, 033001 (2010).

[17] V. Singh, K. S. Hardman, N. Tariq, M.-J. Lu, A. Ellis, M. J. Morrison, and J.D. Weinstein, Phys. Rev. Lett. 108, 203201 (2012).

[18] N. Brahms, T. V. Tscherbul, P. Zhang, J. Klos, R.C. Forrey, Y.S. Au, H. R. Sadeghpour, A. Dalgarno, J. M. Doyle, and T. G. Walker, Phys. Chem. Chem. Phys. 13, 19125 (2011).

[19] W. Behmenburg, A. Makonnen, A. Kaiser, F. Rebentrost, V. Staemmler, M. Jungen, G. Peach, A. Devdariani, S. Tserkovnyi, A. Zagrebin, and E. Czuchaj, J. Phys. B 29, 3891 (1996).

[20] J.-M. Zhu, B.-L. Zhou, and Z.-C. Yan, J. Phys. B 34, 1535 (2001).

[21] J. Elward-Berry and M. J. Berry, J. Chem. Phys. 72, 4500 (1980).

[22] M. Zbiri and C. Daul, J. Chem. Phys. 121, 11625 (2004).

[23] T. R. Roberts and S. G. Sydoriak, Phys. Rev. 102, 304 (1956).

[24] J. E. Sansonetti, W. C. Martin, and S. L. Young, Handbook of Basic Atomic Spectroscopic Data, version 1.1.2 (National Institute of Standards and Technology, Gaithersburg, MD, 2005).

[25] D. Das and V. Natarajan, Phys. Rev. A 75, 052508 (2007).

[26] S. M. Skoff, R. J. Hendricks, C. D. J. Sinclair, J. J. Hudson, D. M. Segal, B. E. Sauer, E. A. Hinds, and M. R. Tarbutt, Phys. Rev. A 83, 023418 (2011).

[27] M.-J. Lu, F. Jose, and J. D. Weinstein, Phys. Rev. A 82, 061802 (2010).

[28] H. Salami and A. J. Ross, J. Mol. Spectrosc. 233, 157 (2005). 
[29] S. Gerstenkorn and P. Luc, Atlas Du Spectre D'Absorption de la Molecule D'Iode (Laboratoire Aime Cotton, CNRS II, Orsay, France, 1978).

[30] D. T. Colbert and W.H. Miller, J. Chem. Phys. 96, 1982 (1992).

[31] J. T. Hougen, The Calculation of Rotational Energy Levels and Rotational Line Intensities in Diatomic Molecules,
Monograph 115 (National Bureau of Standards, Washington, DC, 1970).

[32] J. Brown and A. Carrington, Rotational Spectroscopy of Diatomic Molecules (Cambridge University Press, Cambridge, England, 2003).

[33] H. R. Thorsheim, J. Weiner, and P. S. Julienne, Phys. Rev. Lett. 58, 2420 (1987). 